\title{
Stem Cell Pathways and Notch Signalling an Emerging Role in the Development of Acquired Drug Resistance in Neuroblastoma
}

\author{
John Clark-Corrigal
}

Nursing School of the Ministry of Health

Masood Zaka

National Research Centre

\section{Svetlana Myssina}

University of Sunderland School of Nursing and Health Sciences

\section{Martin Michaelis}

University of Kent Kent Institute of Medicine and Health Sciences: University of Kent

\section{Jindrich Cinatl, Jr}

Goethe-Universität Frankfurt am Main - Campus Bockenheim: Goethe-Universitat Frankfurt am Main

\section{Shafiq Ahmed}

University of Sunderland School of Nursing and Health Sciences

Jane Carr-Wilkinson ( $\boldsymbol{\nabla}$ jane.carr-wilkinson@sunderland.ac.uk)

University of Sunderland https://orcid.org/0000-0001-5030-4262

\section{Primary research}

Keywords: neuroblastoma, stem cells, drug resistance, relapse, bioinformatics, Notch

Posted Date: August 26th, 2021

DOl: https://doi.org/10.21203/rs.3.rs-827092/v1

License: (c) (i) This work is licensed under a Creative Commons Attribution 4.0 International License. Read Full License 


\section{Abstract}

\section{Background}

Neuroblastoma is a paediatric tumour that develops from embryonal neural crest cells that give rise to the sympathetic nervous system. Aggressive high-risk disease remains a clinical challenge and despite multi-modal therapy, survival rates are poor. Most neuroblastomas initially respond well to induction chemotherapy however, $50-60 \%$ of patients with high risk disease will relapse with aggressive disease. A major obstacle in the successful treatment of this disease is the development of acquired resistance to chemotherapeutic agents. We hypothesize that aggressive neuroblastomas acquire a more immature phenotype and an increase in expression stem cell related genes.

Methods

This study investigates the role of stem cell related genes in the development of acquired drug resistance using Q- Real Time PCR and bioinformatics analysis on three-paired vincristine sensitive and resistant cell lines. Results: The study outlines 11 differentially expressed genes with several targets involved in stem cell development, differentiation and the notch signalling pathway.

\section{Conclusions}

The findings implicated in this study, which include comparative analysis against patient microarray data, warrants further study on the functional effects of these genes/pathways to elucidate their role in acquired drug resistance.

\section{Introduction}

Neuroblastoma (NB) is a childhood cancer which affects the developing sympathetic nervous system, specifically the neuronal ganglia which derives from embryonic neural crest cells (1). In the United Kingdom around 90 new cases children under the age of 15 are diagnosed annually (2). High risk patients, categorised by the presence of MYCN amplification or in children over 18 months with metastatic disease, have less than $40 \%$ chance of long term survival (3). Tumour heterogeneity and clonal evolution are now established as characteristic hallmarks of NB, with emerging evidence suggesting that solid tumour cancer cells may not follow the hierarchical organisation of other cancer cells; instead, following clonal evolution from one subtype to another and generating distinctly recognisable tumours. This may explain the variation in clinical manifestations and the unpredictable nature in response to treatment (4). Whilst most high-risk patients do initially respond to induction multimodal chemotherapy, unfortunately over $50 \%$ relapse, and in many cases the returning tumour cells possess a drug resistant phenotype $(5,6)$.

There are a number of chemotherapeutic agents used to treat patients with high risk neuroblastoma including cisplatin, doxorubicin and vincristine. Vincristine (VCR) is a cytotoxic agent which targets cell 
microtubule formation $(7,8)$. Microtubules play integral roles in cell scaffolding as well as being heavily regulated during cell cycle. Thus, microtubule inhibition leads to cell cycle arrest and eventual cell death by apoptosis (9). VCR is currently used in Rapid COJEC induction chemotherapy which comprises a combination of five agents (cisplatin, vincristine, carboplatin, etoposide and cyclophosphamide) for neuroblastoma patients in Europe (10).

A major obstacle in the successful treatment of neuroblastoma is the acquisition of drug resistance to chemotherapeutic agents and hence, is the most pressing factor leading to therapy failure and relapse. Drug resistance is a problem for many cancers including neuroblastoma, with a variety of potential mechanisms driving this resistance including cancer stem cells, epigenetics and genetic alterations(11).

In addition to this, recent studies have shown that neuroblastoma tumours are composed of two distinct cell types; adrenergic (ADRN) and mesenchymal (MES). These cell types are regulated by a super enhancer associated transcription factor network. Interestingly, the MES cells show enhanced migration, heightened chemoresistance and are more prevalent in relapsed disease. Notch 3 signalling is a key driver in the reprogramming of ADRN cells to a more mesenchymal state(12).

Furthermore, epithelial to mesenchymal transition (EMT) has been highlighted as an early mechanism in the development of metastasis. With emerging evidence in literature demonstrating EMT as a causative factor in drug resistance in most cancer types $(13,14)$.

Notch signaling is an evolutionarily conserved signaling pathway that regulates cell fate, including survival and self-renewal of stem cells, proliferation, or differentiation. Deregulation of Notch signaling promotes resistance to cytotoxic therapies by enriching of a small population of resistant cells, referred to as breast cancer stem cells, within the bulk tumor; enhancing stem-like features during the process of dedifferentiation of tumor cells; or promoting epithelial to mesenchymal transition (15). Preclinical studies have shown that targeting the Notch pathway can prevent or reverse resistance through reduction or elimination of breast cancer stem cells. in the central nervous system development in vertebrates, where they have effects on proliferation, apoptosis and lineage decisions (16). This pleiotropic/multifaceted nature is retained during a cancer environment where it has been shown as both an oncogene and a tumour suppressor (17). Dysregulation of Notch signaling, such as activating Notch receptor mutations, overexpression of ligands and/or receptors, and/or overexpression of its target genes, contributes to increased proliferation, cell transformation, and increased drug resistance in cancers of the breast, multiple myeloma, prostate, T-cell acute lymphoblastic leukemia, and others (18)

To further support this mouse models have indeed implicated Notch signaling in the maintenance of neural stem cells within the in the fetal brain. Such observations from developmental biology have shown that Notch exerts similar roles which are vital to the tumourigenicity of cancer stem cells particularly in solid tumors including glioblastoma, ovarian cancer, and breast cancer(17). The cancer stem cell hypothesis suggests that a small distinct subset of undifferentiated cells within the tumour microenvironment possess the ability to regenerate. In addition, these stem cells self-renew and 
differentiate to generate heterogeneous lineages that form the tumour bulk. From this concept comes the theory that the CSCs hold not only the key to drug resistance, but also metastases.

Identification of biological drivers that contribute towards drug resistance, and thus, a means of targeting such drivers, are essential to improving high risk patient survival (19) and are important in order to develop more effective targeted therapeutics. In this study we investigated the potential role for stem cell related genes and pathways, with a special interest in NOTCH signalling, in the development of acquired drug resistance in three neuroblastoma cell lines.

\section{Materials And Methods}

\section{Cell culture}

Three paired parental and drug-adapted neuroblastoma cell lines; SHSY5Y, IMR5 and IMR32 were obtained from the Resistant Cancer Cell Line (RCCL) collection (www.kent.ac.uk/stms/cmp/RCCL/RCCLabout.html). Cell lines were treated with $10 \mathrm{ng} / \mathrm{ml}$ of VCR to generate resistant cells; SHSY5YrVCR, IMR5rVCR, IMR32rVCR. Cells were cultured using Iscove's DMEM (Sigma, UK) with 10\% fetal calf serum (FCS), L- glutamine and1\% primocin. All cell lines were incubated at $37^{\circ} \mathrm{C}, 5 \% \mathrm{CO}$. Cells were passaged once they reached $70-80 \%$ confluency level.

\section{RNA extraction}

RNA extraction was performed using the RNeasy mini kit (Qiagen, uk) according to manufacturer's instructions. RNA quality and quantification were assessed using the Nanodrop Lite Spectrophotometer (Thermo Scientific, UK) and first strand DNA synthesized using the iScript ${ }^{\mathrm{TM}} \mathrm{cDNA}$ synthesis kit (Bio-Rad, UK).

\section{Rt2 Profiler Array}

The Human Cancer Stem Cell RT2 profiler Array analysis (Qiagen, UK) which contains primers for 96 genes related to human cancer stem cells, was performed according to protocol format $\mathrm{R}$ in the manufacturer's instructions on three paired parental and acquired drug resistant cell lines. IMR32 \& IMR32-VCR, IMR5 \& IMR5-VCR and SHSY5Y \& SHSY5Y-VCR. Analysis of mRNA expression levels was performed by Real Time-QPCR (Qiagen Rotorgene 100) of 96 genes related to human cancer stem cells, against a panel of three paired parental and acquired drug resistant cell lines with an acquired drug resistance to the microtubule-inhibitor; Vincristine. Fold change was assessed using the $2 \Delta \Delta \mathrm{ct}$ method, using GAPDH and $\beta$-actin as the respective housekeeping genes.

\section{Bioinformatics analysis}

The CtData for each cell line was further tested using Limma package from R Bioconductor for differential expression of genes by making linear contrast matrices for control and case cell lines. Linear models were then fitted for identification of dysregulation genes using $\operatorname{lm}()$ function from the package. 
Subsequent $p$-values were further tested for multiple testing method of false discovery rate. All genes showing p-values $<0.05$ and $\log 2 \mathrm{FC}>1$ and $<-1$ were picked for overexpression and down regulation. Significant genes from three contrasts were compared for identification of common genes among the cell lines.

\section{Functional analysis}

Functional enrichment analysis were performed on 11 genes using combination of different methods available. Curated gene sets from Molecular Signature Database (MSigDB), DAVID Bioinformatics Resource 6.8, GeneMania and STRING were extracted for the construction of metagene. Fgsea package of Bioconductor was used for the prediction of potential involvement of 11 genes in regulatory processes, functions and pathways. All the pathways with FDR $<0.05$ were considered significant.

\section{In Silico Analysis}

All data was collected from the R2: Genomics Analysis and Visualization Platform 35, using publicly available neuroblastoma patient data. Expression cut-off was determined by a log rank test, which determined an optimum cut off, rather than using an average value. R2: Genomics Analysis and Visualization Platform (http://r2.amc.nl)

Kaplan-Meir plots were constructed to compare high and low expression of the 11 differentially expressed target genes with important clinical parameters stage, overall survival and event free survival. Statistical analysis was shown using a two-tailed, unpaired student t-test with a confidence interval of $95 \%$ ) (Appendix One)

\section{Results}

This study has generated novel data and identified several genes that are up-regulated in drug resistant cell lines; SHSY5YrVCR, IMR5rVCR and IMR32rVCR, compared with the corresponding parental cell line. In addition, we have also identified a subset of genes that are down-regulated in drug resistant cell lines. Many of these candidate genes have been shown to exert a role in aggressive disease in other common cancers such as breast, prostate and small cell lung cancers.

Elevation of stem cell and EMT related genes in chemo-resistant cell lines

Comparative gene expression analysis was undertaken following Real Time-qPCR on each parental and drug resistant cell line. Our results show 11 common genes across the three cell lines, ABCB5, ABCG2, DLL1, ERBB2, ITGA2, LIN28A, LIN28B, MYC, SNAI1, SOX2 and WEE1 (Figure 1A.).

Subsequent unsupervised hierarchical clustering was conducted (Figure 1B.) which observed upregulation of LIN28A, ABCG5, LIN28B and DLL1 in IMR32, with downregulation of ERBB2, ABCG2, WEE1 and MYC. IMR5 displayed upregulation of ITGA2, MYC, SNAI1, WEE1 and ABCG2, with 
downregulation of LIN28B, ABCB5, LIN28A and SOX2. SHSY5Y highlighted upregulation of SOX2, ERBB2, ABCG2 and WEE1, downregulation of SNAI1, DLL1, ITGA2 and MYC (Table 1).

Bioinformatics analysis shows a regulatory network including EMT and stemness genes

In addition to the pathway analysis, we also created a regulatory network composed of 8 of the genes highlighted within our study. This regulatory network developed from connections with previous literature illustrates a core role for MYC, showing direct interactions with WEE1 and SOX2, links through one other gene to ABCG2, ERRBB2, DLL1, ITGA2 and SNAI1 (Figure 2A). In further detail you can see how many genes are influenced by the genes within our network (Figure 2B).

Gene enrichment analysis highlights NOTCH signalling related pathways

Using the 11 target genes, we undertook gene set enrichment analysis; using curated gene sets from a range of different analysis software we discovered substantial links to several notch signalling pathways (Figure 3) highlighting DLL1, MYC and NOTCH1 in particular, all with a highly significant false discovery rate. The Top regulatory pathways extracted from the gene set enrichment analysis were correlated with Significance FDR $<0.05$ this confirmed involvement of the NOTCH signalling pathway in acquired drug resistance (Table 2$)$.

In-silico classification further outlines roles of genes in drug resistance in established studies in other malignancies.

In-silico analysis was performed to determine whether our 11 differentially expressed target genes correlated with important clinical outcomes using four neuroblastoma patient data sets including disease stage, overall survival (OVR) and event free survival (EFS) inpatient data. Kaplan-Meier plots showed that low expression of $A B C B 5, A B C G 2$, and MYC was associated with lower overall survival in patients with stage 4 disease (INSS). Furthermore, elevated expression of ERBB2, ITGA2, LIN28A, LIN28B, SNAI1, SOX2, DLL1 and WEE1 were associated with decreased overall survival in neuroblastoma patients with stage 4 disease (Supplementary figure 1 and table 1).

\section{Discussion}

Neuroblastoma is a paediatric tumour that develops from embryonic neural crest cells that give rise to the sympathetic nervous system. Whilst most neuroblastomas initially respond well to induction chemotherapy, 50-60\% of patients with high risk disease relapse with aggressive disease. Acquired drug resistance provides a major therapeutic challenge in the treatment of such patients.

Using an array of 96 genes related to cancer stem cells in three-paired parental and Vincristine drug resistant neuroblastoma cell lines, this study outlines 11 prominent genes across all cell lines; ABCG2, ERBB2, LIN28A, SOX2, WEE1 MYC, SNAI1, LIN28B, ABCB5, DLL1 and ITAG2 (Fig.1B). Differing expressions of the same genes across the cell lines, highlights the hallmark heterogeneity of disease. 
Gene enrichment analysis, using curated gene sets from a range of different analysis software, highlighted a number of NOTCH signalling pathways; particularly within cancers all with a very significant false discovery rate (Table 1 ). The same genes playing key roles in these regulatory pathways are DLL1, MYC and NOTCH1.

With the help of further bioinformatics analysis, we created a regulatory network composed of 12 genes, 8 of which were highlighted within our study and relating genes discovered from the database (Figure $2 A \& B)$. The network displayed a core role for MYC directly influencing genes associated with oncogenic proteins (ERBB2), self-renewal (SOX2) and cell cycle (WEE1). Indirectly, repression of MYC appears to cause over expression of hallmark drug-resistance genes such as SNAl1 and ABCG2, whilst decreasing expression of NOTCH signalling protein DLL1 and integrin protein ITGA2 (20-22). Interestingly MYC (CMYC) was associated with lower overall survival across four data sets, in contrast to MYCN amplification which is strongly correlated to poor overall and event free survival in high risk neuroblastoma patients (23) suggestive of a potential inverse correlation (24).

Whilst most other cancers display a positive correlation between C-MYC overexpression and a number of the genes highlighted in our study, a number of studies have implicated that c- and MYCN can compensate for loss of expression by one another in normal ESCs, IPSCs and neuroblastoma(25-27). This may explain the findings we have discovered.

Similar to our findings a study in triple negative breast cancer, showed elevated SOX2 expression alongside overexpression of ABCG2 and the EMT marker TWIST1 in drug resistant cells. However, when paclitaxel was administered to SOX2 inhibited mammospheres, ABCG2 and TWIST1 were downregulated, alongside decreased expression of other EMT markers (SNAI1) which induced arrested migration and reduced formation of spheres, indicative of loss of self-renewal (28). This study highlights the potential of the EMT-Stemness axis in therapeutic resistance, where SOX2-dependent TWIST1 overexpression maintains stemness in addition to enabling migration. Subsequent inhibition of the axis causes regained sensitivity.

Furthermore, our study shows deregulation of several Notch signalling pathway genes (Figure 3) suggesting that Notch signalling may play an important role in the development of VCR resistance in neuroblastoma and our cell line models.

EMT is a complex process with different pathways implicated in the process including the Notch signalling pathway. It is now understood that Notch directly influences overexpression of Snail and decreased expression of E-cadherin $(29,30)$. Snai1 expression and loss of E-Cadherin are associated with several cancers and causing increased migration and invasion $(31,32)$, interestingly a recent study in osteosarcoma found that sub-lethal doxorubicin treatments showed significant correlation between EMT and Notch signalling in doxorubicin resistant cell lines(33)which supports our findings within the context acquired drug resistance. In addition, the Notch pathway ligand, Delta like 1 (DLL1), was shown to be associated with disease recurrence in hepatocellular carcinoma (34) and poor prognosis in non-small cell lung cancer (35). A recent study in Neuroblastoma cell lines, showed that DLL1 is highly expressed in 
MYCN amplified cells, and could be inhibited by miRNAs (36). In highly aggressive medulloblastoma, NOTCH signalling pathway has shown to regulate self-renewal and metastasis, with TWIST1 and BMI1 influential in NOTCH1 induced metastases (37). EMT factor TWIST1 has previously been reported in resistance to VCR and other microtubule-disrupting therapeutics (38). Taken together, the EMT-Stemness axis has strong links to both pillars of high-risk disease (therapeutic resistance and metastasis).

Our regulatory network and a wider NOTCH signalling network displayed connections to genes linked to pluripotency including SOX2 and LIN28A \& B. Yamanaka's seminal study on induced pluripotent stem cells (iPSCs) utilised both SOX2 and LIN28A to reprogram somatic cells to an undifferentiated state (39). Since then there has been an emerging interest in genes linked to stem cells and pluripotency and their role in tumorigenesis (40), however whether that role is extended to therapeutic resistance is understudied. The upregulation of both SOX2 and LIN28A across all VCR-resistant cell lines may suggest that the acquisition of stem cell related genes is potentially important for the development of a drugresistant phenotype, which we know occurs in some patients with high risk tumours.

This trend is not limited to VCR resistance alone however, in doxorubicin resistant gastric cancer stem cells, Sox 2 and ABCG2 overexpression was associated with the dox-resistant phenotype (41). Similar can be said for LIN28B overexpression in gastric cancer cells with dox-resistance (42). DLL1 exerts a role in development of drug resistance in dox-resistant cell lines and biopsies in osteosarcoma(43). Interestingly, conflicting studies regarding Snai1 expression in breast adenocarcinoma cell lines in dox-resistant settings $(44,45)$, however Lim et al. 's study does highlight the potential of EMT-stemness interaction in drug resistance. Whilst these results are drawing comparisons across cancers, our data warrants further study into this panel of genes in acquired drug resistance in neuroblastoma.

There have been some studies investigating the relationship between CSC phenotype and EMT (46), where activation of stem cell related pathways directly promote the transition to a mesenchymal profile. Previously, Notch signalling was widely understood as a regulator of cell fate decisions, self - renewal, maintenance of tissue stem cells and tissue wound healing but more recently as a regulator of survival and regeneration of CSCs (47).

However, a definitive molecular mechanism behind the CSC-EMT relationship still remains elusive (13).

Data from in-silico analysis (Supplementary Figure 1 \&Table 1) showed that the worst probabilities for event free survival in late stage (INSS Stage 4) neuroblastoma were ERBB2 overexpression (10\% after 3 years in the Versteeg data set $-88,15 \%$ reach 5 year survival in the Kocak dataset (649)), ITGA2 overexpression ( $0 \%$ were predicted to reach 2 year follow up in both the primary NRC dataset -283 and Versteeg dataset - 88), SOX2 overexpression (10\% at 2 year follow up in the Versteeg dataset, $0 \%$ reached 4 year follow up Kocak dataset (649)) and DLL1 overexpression (predicts $20 \%$ reach 2 year follow up in the Versteeg (88) and NRC (283) ). Confirmation of the link with important clinical parameters in four patient data sets further supports the potential role of these genes in the development of acquired therapeutic resistance in patients with neuroblastoma and potentially other childhood cancers. 
Furthermore, in support of our data a landmark study by van Groningen et al investigating the role of super-enhancers on intra-tumoral heterogeneity identified two distinct lineages; adrenergic (ADRN) and mesenchymal (MES) differentiation with the Notch pathway as a key driver of dedifferentiated mesenchymal identity. In both in vivo and in vitro studies MES cells were shown to be more resistant to common neuroblastoma treatments, which confirms the idea that treatment exerts selective pressure much like the cancer stem cell hypothesis postulates (48). These studies show that ADRN cells can reprogram themselves and become a more dedifferentiated cell type using $\mathrm{NOTCH}$ signalling.

\section{Conclusions}

Our study highlights a core role for MYC and NOTCH signalling associated genes in the development of acquired Vincristine drug resistance in our cell line models. Alterations to individuals within these groups of genes may have further reaching consequences to the stem cell - EMT axis which drive a drug resistant phenotype.

Our panel of three paired Vincristine resistant cell lines provide a unique in-vitro platform to perform studies to understand the molecular mechanisms underlying the development of drug resistance. With the addition of powerful large patient tumour data sets and bioinformatics analyses, we've further highlighted potential for a specific network of genes between niches which have a negative impact on survival and require further research. This could subsequently lead to the discovery of new therapeutic targets that improve the survival of those with the aggressive disease. The findings implicated in this study, which include comparative analysis against microarray data, warrants further studies on the functional effects of the genes and pathways identified in this study in childhood cancers.

\section{Abbreviations}

ABCB5 - ATP-binding cassette sub-family B member 5

ABCG2 - ATP-binding cassette super-family G member 2

ADRN - Adrenergic

BMI1 - Polycomb complex protein BMI-1

CSC - Cancer Stem Cell

DLL1 - Delta like ligand 1

DMEM - Dulbeco's Modified Eagle Medium

DOX - Doxorubicin

EFS - Event Free Survival 
EMT - Epithelial and Mesenchymal Transition

ERBB2 - Erb-B2 Receptor Tyrosine Kinase 2

ESC - Embryonic Stem Cells

FCS - Fetal Calf Serum

IPSCs - Induced Pluripotent Stem Cells

ITGA2 - Integrin alpha 2

LIN28A - LIN 28 homolog A

LIN28B - LIN 28 homolog B

MES - Mesenchymal

MSigDB - Molecular Signature Database

NB - Neuroblastoma

RCS - Resistant Cancer Cell Line

RNA - Ribose Nucleic Acid

RTqPCR - Real Time Quantitative Polymerase Cell Reaction

SNAI1 - Zinc finger protein SNAI1

SOX2 - SRY (sex determining region Y)-box 2

TWIST1 - Twist-related protein 1

VCR - Vincristine

WEE1 - WEE1 G2 Checkpoint Kinase

\section{Declarations}

Ethics approval and consent to participate:

Not applicable

\section{Consent for publication:}

Not applicable 
Availability of data and materials:

The datasets during and/or analysed during the current study available from the corresponding author on reasonable request.

Author Contributions: Conceptualization, Jane Carr-Wilkinson and Shafiq Ahmed.; methodology, Jane Carr-Wilkinson, Martin Michaelis. Jindrich Cinatl, jr Masood Zaka.; software, Masood Zaka.; validation, Svetlana Myssina, Martin Michaelis and John Clark-Corrigall.; formal analysis, Masood Zaka and Svetlana Myssina .; investigation, Masood Zaka and Svetlana Myssina.; resources, , Jane Carr-Wilkinson, Martin Michaelis. Jindrich Cinatl, jr Masood Zaka.; data curation, Masood Zaka.; writing-original draft preparation, Svetlana Myssina, John Clark-Corrigall.; writing-review and editing, Jane Carr-Wilkinson, Shafiq Ahmed.; visualization, Svetlana Myssina, John Clark-Corrigall.; supervision, Jane Carr-Wilkinson, Shafiq Ahmed.; project administration, Jane Carr-Wilkinson.; funding acquisition, Jane Carr-Wilkinson, Shafiq Ahmed.

\section{Consent for publication}

All authors have read and agreed to the published version of the manuscript.

Funding: University of Sunderland - Research Beacon Funding

Acknowledgments: We would like to thank Florian Rothweiler for the administration and shipping of the cell lines used in this study.

Conflicts of Interest: The authors declare no conflict of interest.

\section{References}

1. Louis CU, Shohet JM. Neuroblastoma: Molecular Pathogenesis and Therapy. Annu Rev Med. 2015;66:49-63.

2. Bown N. Neuroblastoma tumour genetics: clinical and biological aspects. J Clin Pathol. 2001 Jan 12;54(12):897-910.

3. Speleman F, Park JR, Henderson TO. Neuroblastoma: A Tough Nut to Crack. Am Soc Clin Oncol Educ Book. 2016;35:e548-557.

4. Ngan ES-W. Heterogeneity of neuroblastoma. Oncoscience. 2015 Aug 24;2(10):837-8.

5. Keshelava N, Zuo JJ, Chen P, Waidyaratne SN, Luna MC, Gomer CJ, et al. Loss of p53 Function Confers High-Level Multidrug Resistance in Neuroblastoma Cell Lines. Cancer Res. 2001 Aug 15;61(16):6185-93. 
6. Owens C, Li BK, Thomas KE, Irwin MS. Surveillance imaging and radiation exposure in the detection of relapsed neuroblastoma. Pediatr Blood Cancer. 2016 Oct;63(10):1786-93.

7. Kotchetkov R, Cinatl J, Blaheta R, Vogel J-U, Karaskova J, Squire J, et al. Development of resistance to vincristine and doxorubicin in neuroblastoma alters malignant properties and induces additional karyotype changes: a preclinical model. Int J Cancer. 2003 Mar 10;104(1):36-43.

8. Piskareva O, Harvey H, Nolan J, Conlon R, Alcock L, Buckley P, et al. The development of cisplatin resistance in neuroblastoma is accompanied by epithelial to mesenchymal transition in vitro. Cancer Lett. 2015 Aug 10;364(2):142-55.

9. Kothari A, Hittelman WN, Chambers TC. Cell cycle-dependent mechanisms underlie vincristineinduced death of primary acute lymphoblastic leukemia cells. Cancer Res. 2016 Jan 1;canres.2104.2015.

10. Smith V, Foster J. High-Risk Neuroblastoma Treatment Review. Children (Basel). 2018 Aug $28 ; 5(9)$.

11. Hanahan D, Weinberg RA. Hallmarks of cancer: the next generation. Cell. $2011 \mathrm{Mar}$ 4;144(5):646-74.

12. Groningen T van, Akogul N, Westerhout EM, Chan A, Hasselt NE, Zwijnenburg DA, et al. A NOTCH feed-forward loop drives reprogramming from adrenergic to mesenchymal state in neuroblastoma. Nature Communications. 2019 Apr 4;10(1):1530.

13. Shibue T, Weinberg RA. EMT, CSCs, and drug resistance: the mechanistic link and clinical implications. Nature Reviews Clinical Oncology. 2017 Oct;14(10):611-29.

14. Tanabe S, Quader S, Cabral H, Ono R. Interplay of EMT and CSC in Cancer and the Potential Therapeutic Strategies. Front Pharmacol [Internet]. 2020 [cited 2021 Mar 10];11. Available from: https://www.frontiersin.org/articles/10.3389/fphar.2020.00904/full

15. Venkatesh V, Nataraj R, Thangaraj GS, Karthikeyan M, Gnanasekaran A, Kaginelli SB, et al. Targeting Notch signalling pathway of cancer stem cells. Stem Cell Investigation [Internet]. 2018 Mar 12 [cited 2021 Feb 14];5(3). Available from: https://sci.amegroups.com/article/view/18689

16. Louvi A, Artavanis-Tsakonas S. Notch signalling in vertebrate neural development. Nature Reviews Neuroscience. 2006 Feb;7(2):93-102.

17. Aster JC, Pear WS, Blacklow SC. The Varied Roles of Notch in Cancer. Annu Rev Pathol Mech Dis. 2017 Jan 24;12(1):245-75.

18. Bolós V, Grego-Bessa J, de la Pompa JL. Notch Signaling in Development and Cancer. Endocrine Reviews. 2007 May 1;28(3):339-63. 
19. Housman G, Byler S, Heerboth S, Lapinska K, Longacre M, Snyder N, et al. Drug resistance in cancer: an overview. Cancers (Basel). 2014 Sep 5;6(3):1769-92.

20. Chen S, Xu Y, Chen Y, Li X, Mou W, Wang L, et al. SOX2 Gene Regulates the Transcriptional Network of Oncogenes and Affects Tumorigenesis of Human Lung Cancer Cells. PLOS ONE. 2012 May 15;7(5):e36326.

21. Ongaratti BR, Silva CBO, Trott G, Haag T, Leães CGS, Ferreira NP, et al. Expression of merlin, NDRG2, ERBB2, and c-MYC in meningiomas: relationship with tumor grade and recurrence. Braz J Med Biol Res [Internet]. 2016 Mar 18 [cited 2019 Oct 19];49(4). Available from:

https://www.ncbi.nlm.nih.gov/pmc/articles/PMC4819410/

22. Kang KW, Im YB, Go W-J, Han H-K. c-Myc Amplification Altered the Gene Expression of ABC- and SLC-Transporters in Human Breast Epithelial Cells. Mol Pharmaceutics. 2009 Apr 6;6(2):627-33.

23. Somasundaram DB, Aravindan S, Yu Z, Jayaraman M, Tran NTB, Li S, et al. Droplet digital PCR as an alternative to FISH for MYCN amplification detection in human neuroblastoma FFPE samples. BMC Cancer. 2019 Jan 28;19(1):106.

24. Westermann F, Muth D, Benner A, Bauer T, Henrich K-O, Oberthuer A, et al. Distinct transcriptional MYCN/c-MYC activities are associated with spontaneous regression or malignant progression in neuroblastomas. Genome Biol. 2008;9(10):R150.

25. Cotterman R, Knoepfler PS. N-Myc Regulates Expression of Pluripotency Genes in Neuroblastoma Including lif, klf2, klf4, and lin28b. PLoS One [Internet]. 2009 Jun 4 [cited 2019 Oct 19];4(6). Available from: https://www.ncbi.nlm.nih.gov/pmc/articles/PMC2686170/

26. Varlakhanova NV, Cotterman RF, deVries WN, Morgan J, Donahue LR, Murray S, et al. myc maintains embryonic stem cell pluripotency and self-renewal. Differentiation. 2010 Jul;80(1):9-19.

27. Nakagawa M, Takizawa N, Narita M, Ichisaka T, Yamanaka S. Promotion of direct reprogramming by transformation-deficient Myc. Proc Natl Acad Sci USA. 2010 Aug 10;107(32):141527.

28. Mukherjee P, Gupta A, Chattopadhyay D, Chatterji U. Modulation of SOX2 expression delineates an end-point for paclitaxel-effectiveness in breast cancer stem cells. Sci Rep [Internet]. 2017 Aug 23;7. Available from: https://www.ncbi.nlm.nih.gov/pmc/articles/PMC5569040/

29. Timmerman LA, Grego-Bessa J, Raya A, Bertrán E, Pérez-Pomares JM, Díez J, et al. Notch promotes epithelial-mesenchymal transition during cardiac development and oncogenic transformation. Genes Dev. 2004 Jan 1;18(1):99-115.

30. Saad S, Stanners SR, Yong R, Tang O, Pollock CA. Notch mediated epithelial to mesenchymal transformation is associated with increased expression of the Snail transcription factor. Int $\mathrm{J}$ Biochem 
Cell Biol. 2010 Jul;42(7):1115-22.

31. Chen J, Imanaka N, Chen J, Griffin JD. Hypoxia potentiates Notch signaling in breast cancer leading to decreased E-cadherin expression and increased cell migration and invasion. British Journal of Cancer. 2010 Jan;102(2):351-60.

32. Zhang L, Sha J, Yang G, Huang X, Bo J, Huang Y. Activation of Notch pathway is linked with epithelial-mesenchymal transition in prostate cancer cells. Cell Cycle. 2017 May 19;16(10):999-1007.

33. Yang J, Guo W, Wang L, Yu L, Mei H, Fang S, et al. Notch signaling is important for epithelialmesenchymal transition induced by low concentrations of doxorubicin in osteosarcoma cell lines. Oncol Lett. 2017 Apr;13(4):2260-8.

34. Ma L, Dong P, Liu L, Gao Q, Duan M, Zhang S, et al. Overexpression of protein Ofucosyltransferase 1 accelerates hepatocellular carcinoma progression via the Notch signaling pathway. Biochem Biophys Res Commun. 2016 Apr 29;473(2):503-10.

35. Pancewicz-Wojtkiewicz J, Eljaszewicz A, Kowalczuk O, Niklinska W, Charkiewicz R, Kozłowski M, et al. Prognostic significance of Notch ligands in patients with non-small cell lung cancer. Oncol Lett. 2017 Jan;13(1):506-10.

36. Bettinsoli P, Ferrari-Toninelli G, Bonini SA, Prandelli C, Memo M. Notch ligand Delta-like 1 as a novel molecular target in childhood neuroblastoma. BMC Cancer [Internet]. 2017 May 19;17. Available from: https://www.ncbi.nlm.nih.gov/pmc/articles/PMC5438559/

37. Kahn SA, Wang X, Nitta RT, Gholamin S, Theruvath J, Hutter G, et al. Notch1 regulates the initiation of metastasis and self-renewal of Group 3 medulloblastoma. Nature Communications. 2018 Oct 8;9(1):4121.

38. Li Q-Q, Xu J-D, Wang W-J, Cao X-X, Chen Q, Tang F, et al. Twist1-Mediated Adriamycin-Induced Epithelial-Mesenchymal Transition Relates to Multidrug Resistance and Invasive Potential in Breast Cancer Cells. Clin Cancer Res. 2009 Apr 15;15(8):2657-65.

39. Takahashi K, Yamanaka S. Induction of Pluripotent Stem Cells from Mouse Embryonic and Adult Fibroblast Cultures by Defined Factors. Cell. 2006 Aug 25;126(4):663-76.

40. Müller M, Hermann PC, Liebau S, Weidgang C, Seufferlein T, Kleger A, et al. The role of pluripotency factors to drive stemness in gastrointestinal cancer. Stem Cell Research. 2016 Mar 1;16(2):349-57.

41. Tian T, Zhang Y, Wang S, Zhou J, Xu S. Sox2 enhances the tumorigenicity and chemoresistance of cancer stem-like cells derived from gastric cancer. J Biomed Res. 2012 Sep;26(5):336-45. 
42. Teng R, Hu Y, Zhou J, Seifer B, Chen Y, Shen J, et al. Overexpression of Lin28 Decreases the Chemosensitivity of Gastric Cancer Cells to Oxaliplatin, Paclitaxel, Doxorubicin, and Fluorouracil in Part via microRNA-107. PLOS ONE. 2015 Dec 4;10(12):e0143716.

43. Pu Y, Zhao F, Wang H, Cai S. MiR-34a-5p promotes multi-chemoresistance of osteosarcoma through down-regulation of the DLL1 gene. Scientific Reports. 2017 Mar 10;7:44218.

44. Lim S, Becker A, Zimmer A, Lu J, Buettner R, Kirfel J. SNAl1-Mediated Epithelial-Mesenchymal Transition Confers Chemoresistance and Cellular Plasticity by Regulating Genes Involved in Cell Death and Stem Cell Maintenance. PLoS One [Internet]. 2013 Jun 17 [cited 2019 Oct 20];8(6). Available from: https://www.ncbi.nlm.nih.gov/pmc/articles/PMC3684605/

45. Tsou S-H, Chen T-M, Hsiao H-T, Chen Y-H. A Critical Dose of Doxorubicin Is Required to Alter the Gene Expression Profiles in MCF-7 Cells Acquiring Multidrug Resistance. PLOS ONE. 2015 Jan 30;10(1):e0116747.

46. Prieto-Vila M, Takahashi R, Usuba W, Kohama I, Ochiya T. Drug Resistance Driven by Cancer Stem Cells and Their Niche. International Journal of Molecular Sciences. 2017 Dec 1;18(12):2574.

47. Capaccione KM, Pine SR. The Notch signaling pathway as a mediator of tumor survival. Carcinogenesis. 2013 Jul 1;34(7):1420-30.

48. Groningen T van, Koster J, Valentijn LJ, Zwijnenburg DA, Akogul N, Hasselt NE, et al. Neuroblastoma is composed of two super-enhancer-associated differentiation states. Nature Genetics. 2017 Aug;49(8):1261-6.

\section{Tables}

Due to technical limitations, table 1 is only available as a download in the Supplemental Files section. Table 2 is not available with this version.

\section{Figures}


A

B

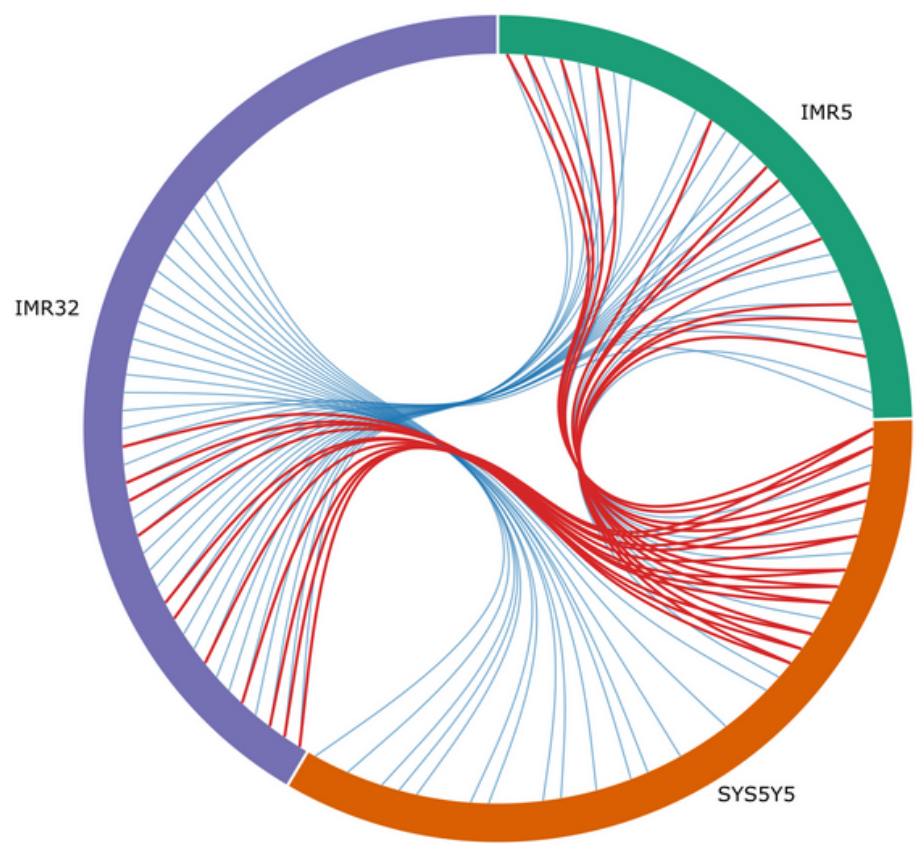

KEY:

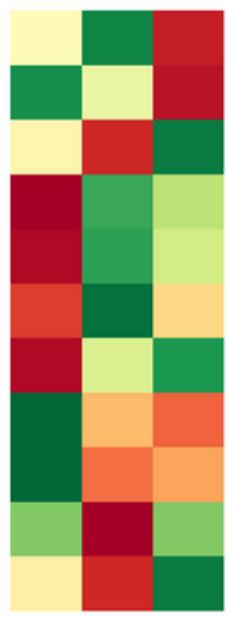

sox 2

ERBB2

ITGA2

LIN28A

ABCB5

LIN28B

DLL1

ABCG2

WEE1

MYC

SNAI1



Up-regulated

Down-regulated Neutral

\section{Figure 1}

Differentially expressed genes identified in three NB cell lines. (A) Venn diagram showing 11 differentially expressed genes among the three cell lines. (B) Heatmap: expression level is colour coded: red for overexpressed and green for under-expressed genes. 
A

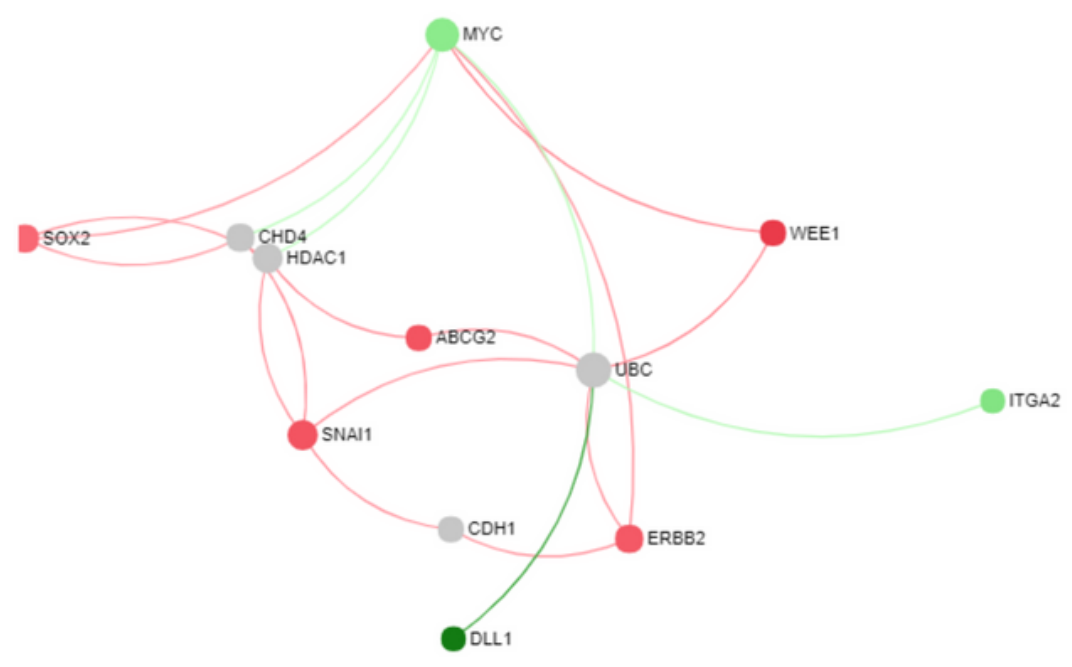

B

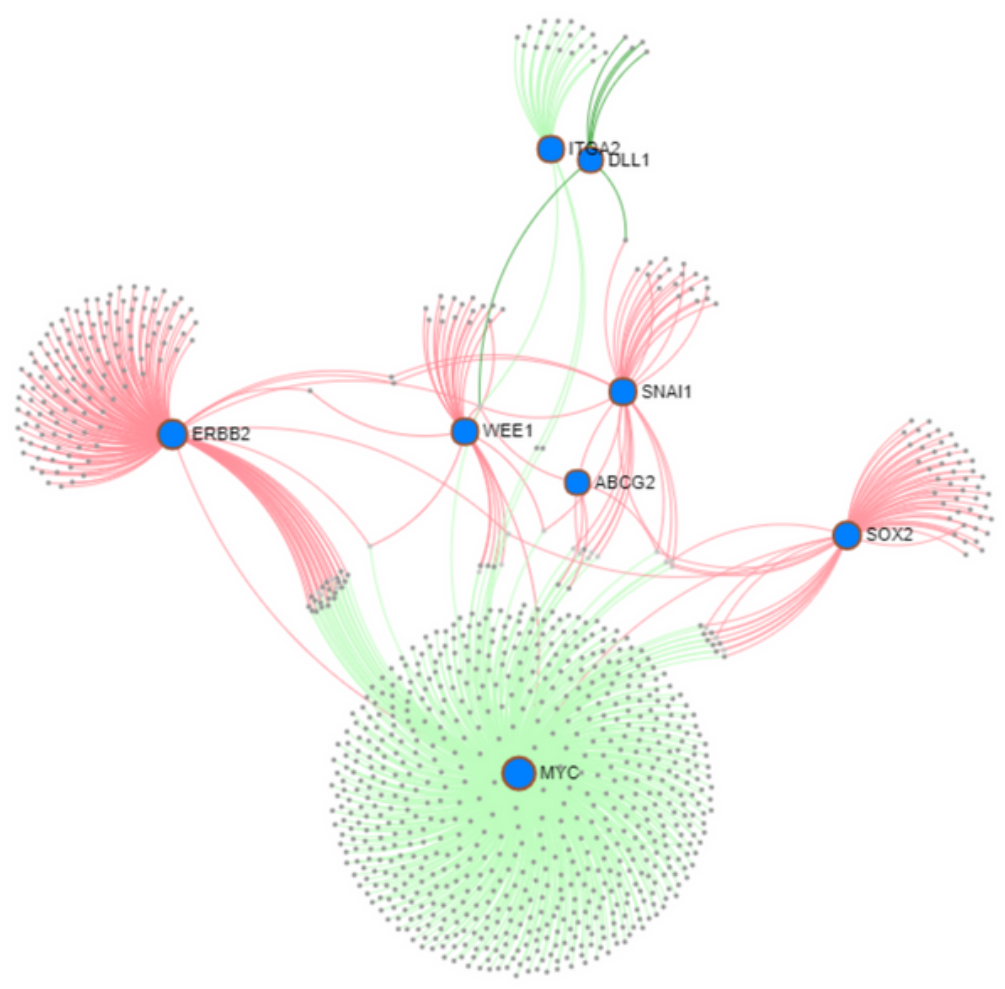

Figure 2

(A) A regulatory network of shared genes. MYC is highlighted in a central role with projections either directly or indirectly to other genes within the study; ABCG2, ERBB2, ITGA2, SNAI1, SOX2 and WEE1. Red colour indicating over-expression of genes and green colour represents under-expression. The grey colour are the connecting genes from database. (B) A regulatory network of shared genes and the effect on 
other genes. Red colour indicating over-expression of genes and green colour represents underexpression. The grey colour are the connecting genes from database.

\section{Figure 3}

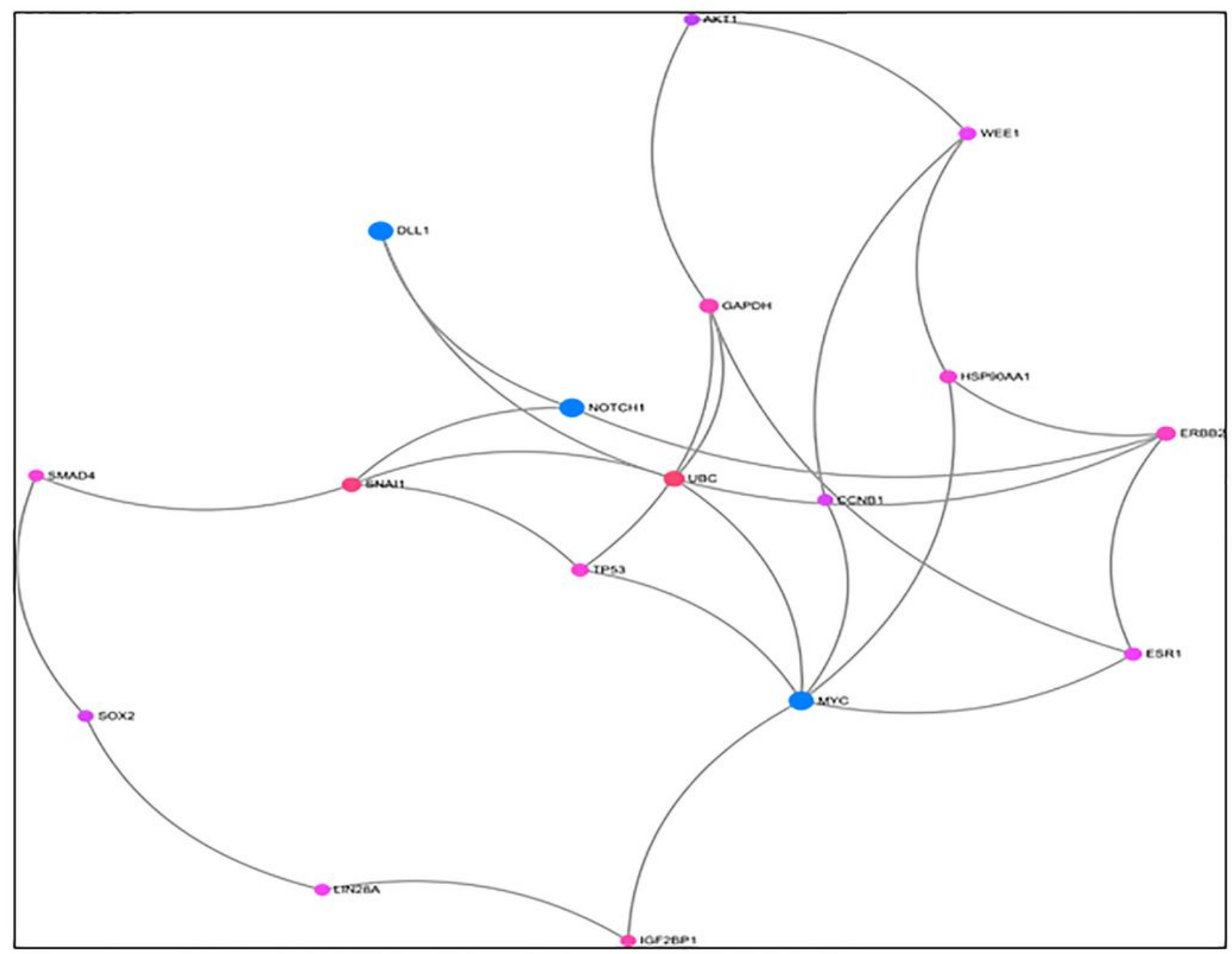

\section{Figure 3}

Network pathway analysis of the NOTCH1 signalling pathway. Genes are highlighted in blue show the main contributors of notch1.

\section{Supplementary Files}

This is a list of supplementary files associated with this preprint. Click to download. 
- Table1JCW180821.pdf

- SupplementaryFiguresmergedAugust2021.pdf 